\title{
Comparison of Antibacterial Effects of Heracleum persicum and Ziziphora tenuior L. Extracts, Calcium Hydroxide and Chlorhexidine on Enterococcus faecalis as Intracanal Medicaments in Root Canal Therapy - An In Vitro Study
}

\author{
Aida Mehdipour ${ }^{1}$, Maryam Akbarzadeh ${ }^{2}$, Somayeh Kermani ${ }^{3}$, Saeed Shams ${ }^{4}$, Alireza Karimi ${ }^{5}$ \\ 1,3,4 Cellular and Molecular Research Center, Qom University of Medical Sciences, Qom, Iran. \\ 2,5 Department of Paediatric Dentistry, Dental School, Qom University of Medical Sciences, Qom, Iran.
}

\section{ABSTRACT}

\section{BACKGROUND}

Given that periapical bacterial infections are considered as one of the causes of endodontic treatment failure, and biomechanical methods may not be able to eliminate bacteria resistant to endodontic treatment, including Enterococcus faecalis; therefore, this study was conducted to compare the antibacterial effects of Golpar and Kakoti extracts with calcium hydroxide and chlorhexidine against $E$. faecalis.

\section{METHODS}

At first, Heracleum persicum (Golpar) and Ziziphora tenuior L. (Kakoti) plants were extracted by ethanol solvent using the cold maceration method and a vacuum distillation apparatus. E. faecalis ATCC 29212 was used as the standard strain in all stages of the experiment. Antibiogram, minimum inhibitory concentration (MIC), and minimum bactericidal concentration (MBC) tests were performed to evaluate the antibacterial activity of the prepared extracts. The anti-biofilm activity of the extracts was evaluated similar to MIC evaluation but with a longer incubation time and analyzed by ELISA at a wavelength of $492 \mathrm{~nm}$.

\section{RESULTS}

The antibiogram test showed a growth inhibition zone diameter of $4 \mathrm{~mm}$ only for chlorhexidine. In addition, chlorhexidine exhibited the highest inhibitory and bactericidal activities in the MIC and MBC tests, respectively (MIC / MBC $<1.562 \mathrm{mg}$ / $\mathrm{mL}$ ). The MIC of Golpar, Kakoti, and Golpar + Kakoti extracts was determined to be $50 \mathrm{mg} / \mathrm{mL}$. No significant inhibitory activity was observed for calcium hydroxide either alone or in combination with the plants' extracts. The MBC of Kakoti extract was $50 \mathrm{mg} / \mathrm{mL}$, and the other compounds had an MBC greater than $100 \mathrm{mg} / \mathrm{mL}$.

\section{CONCLUSIONS}

The present in vitro study showed that the highest inhibitory activity against $E$. faecalis was related to chlorhexidine; however, Golpar and Kakoti extracts at a concentration of $50 \mathrm{mg} / \mathrm{mL}$ exhibited a proper inhibitory activity compared to calcium hydroxide. Therefore, further studies are recommended to be conducted in the future.

\section{KEYWORDS}

Enterococcus faecalis, Ziziphora tenuior L., Heracleum persicum, Chlorhexidine, Calcium Hydroxide, Antibacterial effects.

\author{
Corresponding Author: \\ Saeed Shams, \\ PhD. in Medical Bacteriology, \\ Cellular and Molecular Research Center, \\ Qom University of Medical Sciences, \\ Qom, Iran. \\ E-mail:sshams@muq.ac.ir
}

DOI: $10.14260 /$ jemds/2021/688

How to Cite This Article:

Mehdipour A, Akbarzadeh M, Kermani S, et al. Comparison of antibacterial effects of heracleum persicum and ziziphora tenuior l. extracts, calcium hydroxide, and chlorhexidine on enterococcus faecalis as intracanal medicaments in root canal therapy- an in vitro study. J Evolution Med Dent Sci 2021;10(38):3395-3400, DOI: 10.14260/jemds/2021/688

Submission 23-07-2021, Peer Review 06-09-2021, Acceptance 13-09-2021, Published 20-09-2021.

Copyright (C) 2021 Aida Mehdipour et al. This is an open access article distributed under Creative Commons Attribution License [Attribution 4.0 International (CC BY 4.0)] 


\section{BACKGROUND}

Tooth decay is a progressive disease of hard tooth tissue, which could lead to pulp and periapical disease and intra-root infection if left untreated in the early stages. The pathogenesis of the disease is similar in deciduous and permanent teeth. Reducing or eliminating bacterial infections is one of the most important factors contributing to the successful endodontic (root canal) treatment. ${ }^{1}$ Therefore, the elimination of microorganisms in the root canal before filling it is considered as one of the most important topics in the treatment of tooth root, which is of great importance. ${ }^{2}$ Several studies have shown that Enterococcus faecalis is one of the most common species isolated from the root canal after endodontic therapy. ${ }^{3-5}$ In endodontics, enterococcus species, especially E. faecalis, can lead to unsuccessful root canal treatments, resulting in chronic apical periodontitis. ${ }^{6}$ One of the reasons for the failure of treatment could be due to some virulence factors, such as aggregation substance, lipoteichoic acid, and pheromones, which cause E. faecalis to survive up to $400 \mu \mathrm{m}$ depth in dentinal tubules. ${ }^{7}$

Controlling microbial agents by biomechanical methods could play an important role in successful endodontic treatment; however, there are anatomical variations as well as inaccessible points containing necrotic substances and bacteria that could not be completely cleaned during disinfection. These conditions could act as a good opportunity for bacterial growth, thereby leading to treatment failure in the long run. Therefore, mechanical tools are not enough to kill bacteria due to the complexity of the root canal shape, and the use of intracanal drugs can be helpful.8-11 Due to some side effects of the synthetic materials and safety concerns the interest in natural products has grown now and the most important of these are the products that can be achieved from plants, which have many antimicrobial properties. ${ }^{12}$ Herbal medicines have fewer risks and side effects than chemical medications due to their natural origin as well as closeness and compatibility with the physiology of the human body. Therefore, the use of herbal medicines in the treatment of chronic diseases is more appropriate when used for a long time. In addition, these drugs are cheap and easily available $\mathrm{e}^{13,14}$

Heracleum persicum (Persian hogweed or Golpar) known as angelica in English, is one of the ten species of the genus 3396eracleum in Iran. Golpar is an annual plant that grows in the northern half of the country at altitudes of 1500 meters and above. Golpar extract has been shown to possess antibacterial properties. ${ }^{15}$ Ziziphora tenuior L. (named as Kakoti in Persian), belonging to lamiaceae tribe, lamiales order, and asteridae subclass, is a herbaceous plant that grows in the hillsides. This plant has been shown to have different compounds with various effects, including antibacterial properties. ${ }^{16,17}$ Calcium hydroxide with the chemical formula $\mathrm{Ca}(\mathrm{OH})_{2}$ is one of the most common intracanal medications used in endodontic treatment. Its antimicrobial effect, as well as potential in stimulating the healing of pulp and surrounding tissues, are related to its high alkaline $\mathrm{pH}$. The use of calcium hydroxide has some limitations as it could not remove all microorganisms from the root canal system and requires a long time for exerting its antimicrobial effects. Due to its high $\mathrm{pH}$, this substance is potentially toxic and in the clinical application could lead to soft tissue damage, which in turn could result in chronic inflammation and cell necrosis. Another important limitation in the use of calcium hydroxide is that in the root canal environment, the potential buffering property of dentin reduces the release of hydroxyl ions into the environment, thereby reducing the antimicrobial effect of calcium hydroxide. ${ }^{18}$

Chlorhexidine, as another intracanal medication, has a broad-spectrum antimicrobial activity that could target both categories of bacteria, gram-positive and gram-negative. While chlorhexidine has a significant effect on reducing plaque formation and gingivitis, it is accompanied by several side effects, including staining of the teeth, alteration in taste perception, calculus formation in the oral mucosa, and so on; in addition, it could not dissolve the organic content, necrotic tissue, and smear layer. ${ }^{18-20}$ Calcium hydroxide and chlorhexidine are the most common intracanal medications used in endodontic treatment. Given that herbal medicines such as Kakoti and Golpar extracts have fewer risks and side effects due to their natural origin as well as closeness and compatibility with the physiology of the human body, compared to chemical medicines such as calcium hydroxide and chlorhexidine, ${ }^{21,22}$ this study was performed to evaluate the antibacterial effects of these plants and compare them with calcium hydroxide and chlorhexidine.

\section{METHODS}

This in vitro study was conducted between February 2021 and April 2021.

\section{Preparation of Extracts and Required Substances}

At first, the plants were purchased from a grocery store in Shiraz city, Iran; they were collected from different geographical areas of Shiraz during the 2020 spring and summer seasons. Then $50 \mathrm{~g}$ of aerial parts of Golpar and Kakoti plants were extracted by ethanol solvent using the cold maceration method until the solvent became colourless. The extracts were then concentrated using a vacuum distillation apparatus so that all the solvent was evaporated, the plants pure extracts were prepared and stored in a refrigerator until use. Each of these extracts was used with dimethyl sulfoxide solvent (DMSO, Merck, Germany). The extracts and calcium hydroxide (Golchai Co., Iran) both alone and in combination were prepared and used in the next steps to evaluate the synergistic effect of calcium hydroxide + Kakoti, calcium hydroxide + Golpar, Golpar + Kakoti, and calcium hydroxide + Golpar + Kakoti (in equal volumes).

\section{Preparation of Bacterial Suspension}

A 24-hour culture of the standard strain Enterococcus faecalis ATCC 29212 was prepared, and then a bacterial suspension equivalent to 0.5 McFarland $\left(1.5 \times 10^{8}\right.$ cells / $\left.\mathrm{mL}\right)$ was provided and used in different steps of the study.

\section{Disk Diffusion Method}

In this method, the susceptibility of the bacteria to different compounds was qualitatively evaluated. The bacteria were 
cultured in a spreadsheet on Mueller-Hinton agar medium using a sterile swab. Then wells were created on the culture medium using a Pasteur pipette, and $10 \mu \mathrm{L}$ of the extracts, calcium hydroxide, and the prepared mixtures were added to each well separately and incubated at $37^{\circ} \mathrm{C}$ for 24 hours. Also, $0.2 \%$ chlorhexidine solution (Irannajo Co., Iran) was used as a positive control. The antimicrobial effect of the extracts and the prepared mixtures was determined by measuring the growth inhibition zone diameter around the well containing the desired sample. To quantitatively evaluate the susceptibility of the bacteria, MIC and MBC of the compounds under study were measured.

\section{Minimum Inhibitory and Minimum Bactericidal Concentrations (MIC \& MBC)}

Microdilution method was used to measure MIC. For this purpose, $100 \mu \mathrm{L}$ of Mueller-Hinton broth (Merck-Germany) culture medium was added to the wells of a 96-well plate. The extracts and calcium hydroxide were prepared at a concentration of $200 \mathrm{mg} / \mathrm{mL}$, and then $100 \mu \mathrm{L}$ of each was added to the first well and serially diluted. The final concentrations of each extract were determined as 100,50 , $25,12.5,6.25,3.125$, and $1.562 \mathrm{mg} / \mathrm{mL}$. The culture medium without extract and with bacterial suspension was used as a negative control. Chlorhexidine was also serially diluted and used as a positive control. The plate was incubated at $37^{\circ} \mathrm{C}$ for 24 hours, and then the growth status of the bacteria at different concentrations of the antimicrobial agents was assessed visually. To measure MBC, wells that did not show any bacterial growth in the MIC test were sub-cultured on fresh Mueller-Hinton agar culture medium (Merck, Germany). After incubation at $37{ }^{\circ} \mathrm{C}$ for 24 hours, they were evaluated for growth and colony formation. The minimum concentration of the antimicrobial agents inhibiting the bacterial colony formation was considered as MBC. ${ }^{10}$

\section{Evaluation of Anti-Biofilm Activity}

This test was performed like the MIC test in 96-well polystyrene plates. Incubation time of 72 hours at $37^{\circ} \mathrm{C}$ was considered for biofilm formation. After incubation, the contents of the plates were emptied, and the wells were washed three times with sterile saline solution. To stabilize the formed biofilms, $200 \mu \mathrm{L}$ of methanol was added to the wells, and the plates were placed at room temperature for 15 minutes. After removing the alcohol and drying the plates, $200 \mu \mathrm{L}$ of $1 \%$ crystal violet dye was added to each well, and the plates were again placed at room temperature for 20 minutes. The plates were then washed with sterile saline solution to remove excess dye. The dye attached to the biofilm was released with $200 \mu \mathrm{L}$ of methanol and finally read at $492 \mathrm{~nm}$ using an ELISA reader. All the experiments mentioned above were performed in triplicate.

\section{Statistical Analysis}

In this study, non-parametric methods were used to evaluate the results. Statistical analysis was performed by Excel software.

\section{RESULTS}

The antibiogram test showed a growth inhibition zone diameter of $4 \mathrm{~mm}$ only for chlorhexidine (Figure 1).
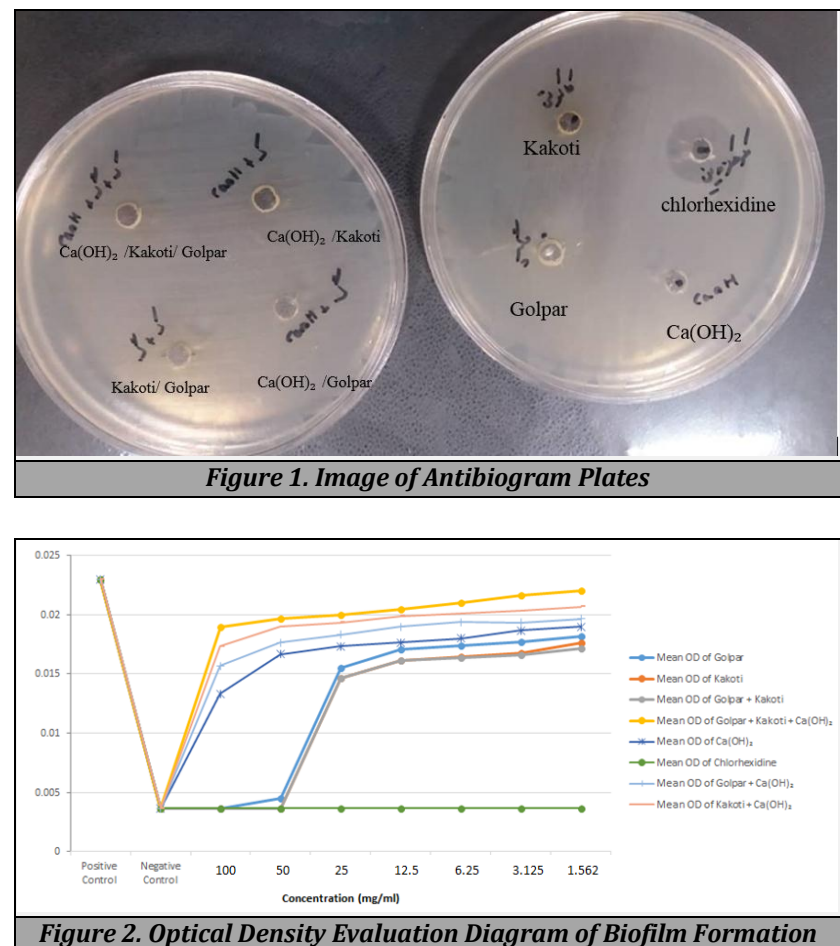

\begin{tabular}{|c|c|c|c|c|c|c|c|c|}
\hline & 흥 & 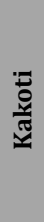 & 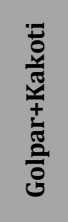 & 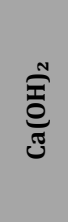 & 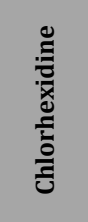 & 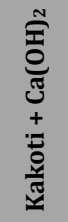 & 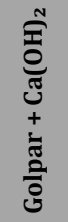 & 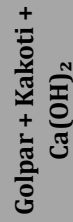 \\
\hline $\begin{array}{c}\text { MIC } \\
(\mathrm{mg} / \mathrm{mL})\end{array}$ & 50 & 50 & 50 & $>100$ & $<1.562$ & $>100$ & $>100$ & $>100$ \\
\hline $\begin{array}{c}\mathrm{MBC} \\
(\mathrm{mg} / \mathrm{mL})\end{array}$ & $>100$ & 50 & $>100$ & $>100$ & $<1.562$ & $>100$ & $>100$ & $>100$ \\
\hline $\begin{array}{c}\text { Antibiogram } \\
(\mathrm{mm})\end{array}$ & 0 & 0 & 0 & 0 & 4 & 0 & 0 & 0 \\
\hline Antibiofilm & ++ & ++ & ++ & + & ++++ & - & - & - \\
\hline \multicolumn{9}{|c|}{$\begin{array}{l}\text { Table 1. Comparison of MIC, MBC, Antibiogram, and Anti-Biofilm } \\
\text { Activity of the Compounds Evaluated in this Study }\end{array}$} \\
\hline
\end{tabular}

In the MIC test, chlorhexidine exhibited the highest inhibitory activity so that no bacteria could grow in its vicinity even at a low concentration of $1.562 \mathrm{mg} / \mathrm{mL}$. The MIC of Golpar, Kakoti, and Golpar + Kakoti extracts was determined to be $50 \mathrm{mg} / \mathrm{mL}$. No significant inhibitory activity was observed for calcium hydroxide either alone or in combination with the plants' extracts. The MBC test results showed that, in addition to chlorhexidine as control which caused no bacterial colonies to form on the plate even at low concentrations, only the MBC of Kakoti was $50 \mathrm{mg} / \mathrm{mL}$, and the other compounds had an MBC greater than $100 \mathrm{mg} / \mathrm{mL}$.

Figure 2 shows different concentrations of the compounds and their optical densities (OD). As shown in the figure, the highest OD was related to the positive control due to the high bacterial growth rate, more biofilm formation, and more dye absorption, while the lowest OD was related to the negative control due to no bacterial growth, no biofilm formation, and no dye absorption. The biofilm formation and 
OD in wells containing chlorhexidine were equivalent to the negative control. The anti-biofilm activity of Golpar, Kakoti, and their combination was similar, and no biofilm was formed at concentrations of 100 and $50 \mathrm{mg} / \mathrm{mL}$. After Golpar and Kakoti, the next compound able to inhibit biofilm formation was calcium hydroxide alone. The other compounds formulated with calcium hydroxide showed no inhibitory activity against biofilm formation. More information is presented in Table 1.

\section{DISCUSSION}

Tooth decay is a progressive disease of hard tooth tissue, which could lead to pulp and periapical disease and intra-root infection if left untreated in the early stages. The pathogenesis of the disease is similar in deciduous and permanent teeth. Reducing or eliminating bacterial infections is one of the most important factors contributing to successful endodontic treatment. ${ }^{1}$ Bacteria and their products may be the cause of endodontic treatment failure, and their persistent presence in apparently well-treated root canals may disrupt postoperative healing processes. Several studies have shown that $E$. faecalis is one of the most common species isolated from the root canals after endodontic treatment. Enterococci are usually resistant to various intracanal treatment procedures. ${ }^{3,5}$

Control of microbial agents by biomechanical methods could play an important role in successful root canal treatment. But biomechanical preparation of root canal does not eliminate bacteria, and the use of root canal medications could be more effective. ${ }^{9}$ The use of medicinal plants for therapeutic purposes has a long history. Despite the considerable amount of antibiotics that have been produced naturally or semi-synthetically from natural products and introduced to the market, the antimicrobial activity of about $20 \%$ of plants in the world have been tested and approved pharmacologically and biologically.

Golpar plant has many properties due to different compounds such as Octyl 2-methyl butyrate, Pentylcyclopropane, Octyl isobutyrate, etc. This plant has various applications in traditional medicine, and its antibacterial activity against various bacteria has been well documented, including Escherichia coli, Staphylococcus aureus, Listeria monocytogenes, etc. ${ }^{23}$

Kakoti plant also has antimicrobial effects due to different compounds. The main compounds in some plants of the lamiaceae family, including Kakoti, are pulegone and isomenthone. This plant has been used for centuries in traditional medicine to treat various medical conditions. ${ }^{16,17,24}$ Calcium hydroxide is commonly used in endodontics as an intracanal medication to eliminate microorganisms during chemomechanical preparation. 25

The antibiogram test in the present study showed a growth inhibition zone diameter of $4 \mathrm{~mm}$ only for chlorhexidine, while the antibacterial effect of the other compounds was not detectable. This finding was inconsistent with the finding of a study by Nazemisalman et al. (2018). In their study, according to the mean diameter of the growth inhibition zone, the antibacterial activity of $Z$. tenuior was shown to be higher than that of chlorhexidine, ${ }^{17}$ while the
MIC test results in the present study showed that the highest antimicrobial activity was related to chlorhexidine, followed by Golpar, Kakoti, and their combination. In the MBC test, in addition to chlorhexidine which caused no bacterial colonies to form on the plate, only the bactericidal activity of Kakoti at a concentration of $50 \mathrm{mg} / \mathrm{mL}$ was significant.

Nazemisalman et al. also investigated the antibacterial effects of Ziziphora tenuior, Dracocephalum moldavica, Ferula gummosa, and Prangos ferulacea essential oils against E. faecalis strains. Their study results showed that all four essential oils had antibacterial activity against E. faecalis, and Kakoti exhibited the highest antibacterial activity. ${ }^{17}$ These findings are consistent with the findings of the present study.

The antibacterial effect of $H$. persicum essential oil against some food borne bacteria including S. aureus, Salmonella enterica, E. coli, Vibrio cholera, and Yersinia enterocolitica was investigated by Mostaghim et al. According to the results, this extract exhibited good antibacterial activity against the bacteria. ${ }^{26}$

Salehi et al. (2005) investigated the antibacterial and antioxidant properties of $Z$. clinopodioides subsp. rigida against seven bacterial species including E. coli, $S$. aureus, $E$. faecalis, etc. The results showed that the Kakoti plant had good antibacterial activity against $E$. faecalis strains (MIC $>15$ $\mathrm{mg} / \mathrm{mL})^{27}$

The antimicrobial properties of Persian hogweed $(H$. persicum) extract against 14 bacterial and two fungal species were investigated in vitro by Nazemi et al. (2005). Aqueous extract of $H$. persicum showed no significant antimicrobial activity; however, its methanolic extract had a good antimicrobial effect against bacillus, streptococcus, enterococcus, and nocardia, ${ }^{15}$ these findings are consistent with the findings of the present study.

In this study, chlorhexidine exhibited the highest antibiofilm activity against the target bacterium, followed by Golpar and Kakoti extracts and their combination; calcium hydroxide alone was the next compound able to inhibit biofilm formation. The other compounds formulated with calcium hydroxide had no significant inhibitory activity against biofilm formation.

The antibacterial effects of herbal and synthetic medications against E. faecalis were investigated by Kalaiselvam et al. (2019). They examined the antimicrobial effect of six different compounds on tooth intracanal disinfection. In their study, $2 \%$ chlorhexidine gel exhibited the highest antibacterial activity, followed by calcium hydroxide, ginger, black pepper, long pepper, and saline. ${ }^{9}$

Eswar et al. compared the effectiveness of garlic extract with $2 \%$ chlorhexidine and calcium hydroxide in disinfecting root canals infected with E. faecalis. According to their study results, $2 \%$ chlorhexidine had the highest antibacterial effect against the target bacterium compared to garlic extract and calcium hydroxide. ${ }^{3}$

In another study by Attia et al. (2015), the antibacterial effects of three different compounds, including calcium hydroxide paste $(\mathrm{CaOH})$, chlorhexidine gluconate $(\mathrm{CHX})$ gel, and antibiotic-corticosteroid paste, against E. faecalis, $S$. mutans, and Candida albicans were investigated. In their study, chlorhexidine exhibited the highest antibacterial effect against the three bacteria studied. ${ }^{28}$ 


\section{CONCLUSIONS}

In the present study, the highest inhibitory effect was related to chlorhexidine; however, Golpar and Kakoti extracts and their combination also exhibited a proper inhibitory activity. No significant inhibitory activity was observed for calcium hydroxide either alone or in combination with the plants' extracts. Therefore, after further studies, these extracts may be used as a suitable mouthwash to prevent tooth decay.

\section{Acknowledgment}

This project was taken from a dental dissertation with the ethics code IR.MUQ.REC.1399.110. Hereby the authors would like to thank the Vice Chancellor for Research of Qom University of Medical Sciences for financial support.

Data sharing statement provided by the authors is available with the full text of this article at jemds.com.

Financial or other competing interests: None.

Disclosure forms provided by the authors are available with the full text of this article at jemds.com.

\section{REFERENCES}

[1] Dutta B, Dhull KS, Das D, et al. Evaluation of antimicrobial efficacy of various intracanal medicaments in primary teeth: an in vivo study. International Journal of Clinical Pediatric Dentistry 2017;10(3):267-71.

[2] Kazemizadeh Z, Tashakori M, Rezaeian M. Comparison of the antimicrobial effect of garlic extract with two intracanal irrigants on enterococcus faecalis. Journal of Rafsanjan University of Medical Sciences 2011;10(1):313.

[3] Eswar K, Venkateshbabu N, Rajeswari K, et al. Dentinal tubule disinfection with $2 \%$ chlorhexidine, garlic extract and calcium hydroxide against Enterococcus faecalis by using real-time polymerase chain reaction: In vitro study. Journal of Conservative Dentistry 2013;16(3):194-8.

[4] Parolia A, Kumar H, Ramamurthy S, et al. Effect of propolis nanoparticles against enterococcus faecalis biofilm in the root canal. Molecules 2021;26(3):715.

[5] Revati S, Bipin C, Chitra PB, et al. In vitro antibacterial activity of seven Indian spices against high level gentamicin resistant strains of enterococci. Archives of Medical Science 2015;11(4):863-8.

[6] Arora S, Saquib SA, Algarni YA, et al. Synergistic effect of plant extracts on endodontic pathogens isolated from teeth with root canal treatment failure: an in vitro study. Antibiotics (Basel) 2021;10(5):552.

[7] Mavani HAK, Tew IM, Wong L, et al. Antimicrobial efficacy of fruit peels eco-enzyme against enterococcus faecalis: an in vitro study. International Journal of Environmental Research and Public Health 2020;17(14):5107.

[8] Jafari A, Heidari A, Kiyani $M$, et al. Evaluation of antimicrobial effect of chlorhexidine and two different concentrations of thyme essence in pulp therapy of deciduous teeth. Iranian Journal of Pediatric Dentistry 2015;10(2):35-44.
[9] Kalaiselvam R, Soundararajan K, MathanRajan R, et al. Comparative evaluation of the anti-bacterial efficacy of herbal medicaments and synthetic medicaments against enterococcus faecalis using real-time polymerase chain reaction. Cureus 2019;11(7):e5228.

[10] Parham S, Kharazi AZ, Bakhsheshi-Rad HR, et al. Antioxidant, antimicrobial and antiviral properties of herbal materials. Antioxidants 2020;9(12):1309.

[11] Siddique R, Ranjan M, Jose J, et al. Clinical quantitative antibacterial potency of garlic-lemon against sodium hypochlorite in infected root canals: a double-blinded, randomized, controlled clinical trial. Journal of International Society of Preventive \& Community Dentistry 2020;10(6):771-8.

[12] Nagy-Bota MC, Man A, Santacroce L, et al. Essential oils as alternatives for root-canal treatment and infection control against enterococcus faecalis-a preliminary study. Applied Sciences 2021;11(4):1422.

[13] Nikseresht R, Shams S, Mehdipour A, et al. Evaluation of the antibacterial effects of three natural formulated mouthwashes against Streptococcus mutans: an in vitro study. Journal of Oral Health and Oral Epidemiology 2019;8(2):97-103.

[14] Pakdel F, Ghasemi S, Babaloo A, et al. Antibacterial effects of garlic extracts and ziziphora essential oil on bacteria associated with peri-implantitis. Journal of Clinical and Diagnostic Research 2017;11(4):ZC16-ZC19.

[15] Nazemi A, Khataminezhad M, Pourshamsian K, et al. Antimicrobial activity of aqueous and methanol extracts of Heracoleum Persicum. Medical Sciences 2005;15(2):91-4.

[16] Najafi F, Tavakkoli Z. Comparing essential oil composition and antibacterial effects of Ziziphora tenuior L. in two regions of Iran. Iranian Journal of Medical and Aromatic Plants 2011;27:239-48.

[17] Nazemisalman B, Vahabi S, Yazdinejad A, et al. Comparison of antimicrobial effect of Ziziphora tenuior, Dracocephalum moldavica, Ferula gummosa and Prangos ferulacea essential oil with chlorhexidine on Enterococcus faecalis: an in vitro study. Dental Research Journal (Isfahan) 2018;15(2):111-16.

[18] Adel M, Sharifi M, Hamedi R, et al. Comparing the antimicrobial efficacy of carvacrol, chlorhexidine and calcium hydroxide on enterococcus faecalis in root canal treatment: an in vitro study. Journal of Mazandaran University of Medical Sciences 2016;26(143):108-19.

[19] Flötra L, Gjermo P, Rölla G, et al. Side effects of chlorhexidine mouth washes. Scand J of Dent Res 1971;79(2):119-25.

[20] Sinha DJ, Nandha KD, Jaiswal N, et al. Antibacterial effect of Azadirachta Indica (Neem) or Curcuma longa (Turmeric) against Enterococcus faecalis compared with that of $5 \%$ sodium hypochlorite or $2 \%$ chlorhexidine in vitro. The Bulletin of Tokyo Dental College 2017;58(2):103-9.

[21] Ahmady-Asbchin S, Omran NA, Jafari N, et al. Antibacterial effects of Lavandula Stoechas essential oil, on gram positive and negative bacteria. Medical Laboratory Journal 2012;6(2):35-41.

[22] Izadi Z, Sorooshzadeh A, Sanavi SAMM, et al. Investigation on antimicrobial effects of essential oil of purple coneflower (Echinacea Purpurea L.) and 
identification of its chemical compounds. Iran South Med Journal 2014;17(1):58-69.

[23] Rezayan A, Ehsani A. Evaluation of the chemical compounds and antibacterial properties of the aerial parts of persian Heracleum persicum essence. Journal of Babol University of Medical Sciences 2015;17(6):26-32.

[24] Aghajani Z, Assadian F, Masoudi S, et al. Chemical composition and in vitro antibacterial activities of the oil of Ziziphora clinopodioides and Z. capitata subsp. capitata from Iran. Chemistry of Natural Compounds 2008;44(3):387-9.

[25] Kim D, Kim E. Antimicrobial effect of calcium hydroxide as an intracanal medicament in root canal treatment: a literature review: Part I. In vitro studies. Restorative Dentistry \& Endodontics 2014;39(4):241-52.
[26] Shariatifar N, Mostaghim T, Afshar A, et al. Antibacterial properties of essential oil of Heracleum persicum (Golpar) and foodborne pathogens. Int J Enteric Pathog 2017;5(2):41-4.

[27] Salehi P, Sonboli A, Eftekhar F, et al. Essential oil composition, antibacterial and antioxidant activity of the oil and various extracts of Ziziphora clinopodioides subsp. rigida (BOISS.) R ECH. f. from Iran. Biological and Pharmaceutical Bulletin 2005;28(10):1892-6.

[28] Attia D, Farag A, Afifi I, et al. Antimicrobial effect of different intracanal medications on various microorganisms. Tanta Dental Journal 2015;12(1):41-7. 